\title{
XIV. On the distribution of energy in a mass of liquid in a state of steady motion
}

\author{
James H. Cotterill M.A.
}

To cite this article: James H. Cotterill M.A. (1876) XIV. On the distribution of energy in a mass of liquid in a state of steady motion, Philosophical Magazine Series 5, 1:2, 108-111, DOI: 10.1080/14786447608639011

To link to this article: http://dx.doi.org/10.1080/14786447608639011

曲 Published online: 13 May 2009.

Submit your article to this journal $[\pi$

Џ Article views: 3

Q View related articles $₫$ 


\section{Prof. J. H. Cotterill on the Distribution of Energy}

plane. The deflections became now considerably greater, and, besides, corresponded perfectly with those obtained with the magnetometer.

The two mutually independent methods of investigation have thus conducted us to the same result, that foretold by the theory -namely, that the resistance to galvanic conduction is diminished when the conductor and the current move in the same direction, but is increased when the directions are opposite.

I will only mention, in conclusion, that I have tried to employ for this investigation a saturated solution of sulphate of copper, with copper poles, and a solution of sulphate of zinc, with amalgamated zinc poles; but these liquids were quite inapplicable, because 1 could never succeed in rendering the polar plates galvanically so equal that, when the plates dipped into the liquid, a slight current would not arise, which immediately varied as soon as the liquid was set in motion. Whence this originated I cannot say. The sulphate-of-zinc solution, however, contained some iron.

XIV. On the Distribution of Energy in a mass of Liquid in a state of Steady Motion. By James H. CotTerill, M.A., Professor of Applied Mechanics in the Royal Naval College, Greenwich *.

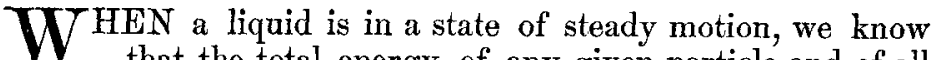
that the total energy of any given particle and of all that follow it in one and the same line of motion is a constant quantity ; or, as we may otherwise express it, the total energy of each elementary stream is uniformly distributed. I am not, however, aware that it has been hitherto noticed that the distribution of energy among the several elementary streams, of which the whole current is supposed to be made up, depends solely on the molecular rotation at each point of the liquid, and is uniform when the motion is irrotational.

For simplicity suppose the motion to be in two dimensions, and to take place either in a vertical or horizontal plane. In the first case the liquid will be under the action of gravity only; and in the second, which will be included in the first, the effect of gravity need not be considered. In the figure, let $A B, C D$ be consecutive lines of motion, and $P Q$ a normal to these lines, and let $\mathrm{P}$ and $\mathrm{Q}$ be particles moving in these lines. I Then, if $h$ be the total bead at $Q$ (that is, the total energy of $Q$ per unit of weight),

$$
h=z+\frac{p}{w}+\frac{v^{2}}{2 g}
$$

* Communicated by the Author. 
in a mass of Liquid in a state of Steady Motion.

where $z$ is the elevation of $\mathrm{Q}$ above some given datum level, and $w$ is the weight of a cubic foot of water. Differentiating,

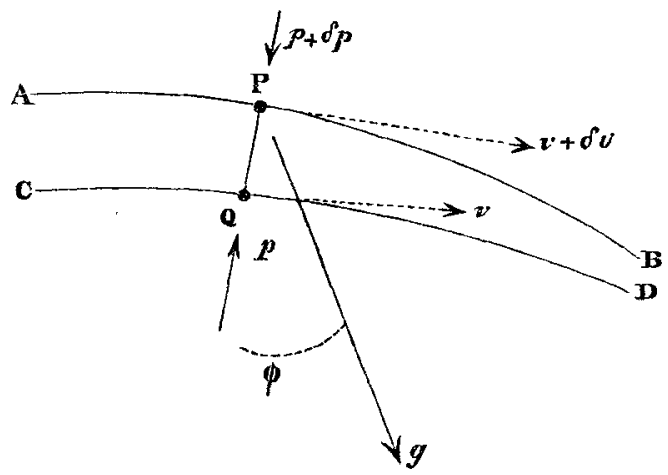

we get

$$
\delta h=\delta z+\frac{\delta p}{w}+\frac{v \delta v}{g}
$$

for the head at $\mathrm{P}$, where $\delta z$, being the elevation of $\mathrm{P}$ above $\mathrm{Q}$, is given by

$$
\delta z=\mathrm{PQ} \cdot \cos \phi,
$$

where $\phi$ is the angle $P Q$ makes with the vertical.

But if we imagine a small cylinder described round $P Q$ as an axis and consider its equilibrium, it is clear that

$$
\delta p \cdot \alpha=\frac{w}{g} \cdot \frac{v^{2}}{p} \alpha \cdot \mathrm{PQ}-w \cdot \alpha \cdot \mathrm{PQ} \cdot \cos \phi,
$$

where $\alpha$ is the sectional area of the cylinder, and $\rho$ the radius of curvature of the lines of motion at $\mathrm{PQ}$. Combining this with the former equation, we get

$$
\delta h=\frac{v^{2}}{g \rho} \cdot \mathrm{PQ}+\frac{v \cdot \delta v}{g}=\frac{v \cdot \mathrm{PQ}}{g}\left\{\frac{v}{\rho}+\frac{\delta v}{\mathrm{PQ}}\right\} .
$$

Now it is already known that, if through a given particle $A$ lines be drawn through $B$ and $C$, two particles very near to $A$, such that $A B$ and $B C$ are at right angles to each other at the instant considered, then the mean angular velocity of these lines is the same in whatever direction they be drawn through $A$, and is equal to the angular velocity with which a small cylindrical element described round A would rotate if supposed suddenly solidified, which mean angular velocity may hence conveniently be called the molecular rotation. In the present case $\frac{v}{\rho}$ is the angular velocity of the tangent, that is, of a line 
drawn through two consecutive moving particles; and $\frac{\delta v}{\mathrm{PQ}}$ is the angular velocity reckoned in the same direction of a line drawn perpendicular to the tangent through $P$ and $Q$, two consecutive moving particles. The sum of the two is therefore twice the molecular rotation; and if we call the molecular rotation $\cup$, we shall have

$$
\delta h=2 \cdot \frac{v \cdot \mathrm{PQ}}{g} \cdot \bullet \cdot \cdot \cdot \cdot \cdot \cdot
$$

Now $v . \mathrm{PQ}$ is constant, being the flow in an elementary stream of breadth unity; and hence we see that the difference of energy between two consecutive elementary streams is proportional to the molecular rotation at any point of either.

An immediate consequence of this is that the molecular rotation is the same at all points situated in a line of motion, and can be determined when the difference of head is known. Thus in the well-known trochoidal motion, the difference of head between one trochoidal layer and the next consecutive is easily seen to be $\frac{2 r}{\mathrm{R}}$. $\delta r$, where $r$ is the length of the tracing-arm, and $\mathrm{R}$ the radius of the rolling circle. Hence, placing this in equation (1), the known value of the molecular rotation is more easily determined than in any other way.

Again, we learn that if the energy of any portion of the liquid be initially uniformly distributed, the motion of that portion must be irrotational and must always remain so ; and thus we have a simple demonstration that the permanent motion of a perfect liquid past a solid free from discontinuity of curvature and perfectly smooth is necessarily irrotational. This demonstration clearly also applies to any case in which a perfectly smooth and fair-formed solid moves with a uniform motion of translation through a liquid at rest.

The peculiar form in which the condition for irrotational motion is expressed, namely

$$
\frac{v}{\rho}+\frac{\delta v}{\mathrm{PQ}}=0
$$

leads to a conclusion of much importance-namely, that by a surface of "fair" form is to be understood a surface free from discontinuity of curvature, and not merely from discontinuity of form : thus a circular arc joined to a straight line is not a fair form, since the radius of curvature changes abruptly and occasions a discontinuity of form in the next consecutive 
stream-line. This, of course, is also true when the molecular rotation is not zero, but any constant.

When the motion is not confined to one plane, the energy of the fluid will be uniformly distributed in any case in which the molecular rotation is zero, or more generally when the axis of rotation of a fluid element is likewise the direction of its motion of translation; but the consideration of this kind of motion is postponed for the present.

\section{The History of Young's Discovery of his Theory of Colours. By Alffred M. Ma Yer.}

To the Editors of the Philosophical Magazine and Journal.

Gentlemen,

Stevens Institute of Technology, Hoboken, New Jersey, December 13, 1875.

I HAVE the honour to send you for publication in your Journal the accompanying paper, entitled "The History of Young's Discovery of his Theory of Colours." Disjointed extracts from this paper have appeared in English journals; and I am therefore desirous that the entire paper should appear in your Journal, if you should deem it worthy of that distinction.

$$
\begin{aligned}
& \text { I remain, Gentlemen, } \\
& \text { Very respectfully yours, } \\
& \text { ALFRED M. MAYER. }
\end{aligned}
$$

The object of this communication is twofold : I desire first to give complete abstracts from the writings of Newton, Young, and Wollaston, in order to put the student of science in possession of all of the early literature relating to Young's theory of colours ; in the second place, I propose to trace the curious history of the steps by which Young was led to the final adoption of what is now known as Young's theory of colour-sensation. In accomplishing the first of these objects, I shall at the same time attempt to show, 1st, that Young first formed an hypothesis similar to that known as Brewster's (that is, he selected red, yellow, and blue as the three simple colour-sensations); 2nd, that he subsequently modified his hypothesis and adopted red, green, and violet as the three elementary colour-sensations, showing that up to the date of this change of opinion all of his ideas on the subject were bypothetical, and not based on any observations or experiments of his own or of others; $3 \mathrm{rd}$, that this change of opinion as to the three elementary colours was made on the basis of a misconception by Wollaston of the nature of his celebrated obser- 